\title{
The speed of light and the Hubble parameter: the Mass-Boom effect
}

\author{
Antonio Alfonso-Faus
}

\begin{abstract}
We prove here that Newton's universal gravitation and momentum conservation laws together reproduce Weinberg's relation. It is shown that the Hubble parameter $H$ must be built in this relation, or equivalently the age of the Universe $t$. Using a wave-to-particle interaction technique we then prove that the speed of light $c$ decreases with cosmological time, and that $c$ is proportional to the Hubble parameter $H$. We see the expansion of the Universe as a local effect due to the $\mathrm{LAB}$ value of the speed of light $c_{0}$ taken as constant. We present a generalized red shift law and find a predicted acceleration for photons that agrees well with the result from Pioneer 10/11 anomalous acceleration. We finally present a cosmological model coherent with the above results that we call the Mass-Boom. It has a linear increase of mass $m$ with time as a result of the speed of light $c$ linear decrease with time, and the conservation of momentum $m c$. We obtain the baryonic mass parameter equal to the curvature parameter, $\Omega_{m}=\Omega_{k}$, so that the model is of the type of the Einstein static, closed, finite, spherical, unlimited, with zero cosmological constant. This model is the cosmological view as seen by photons, neutrinos, tachyons etc. in contrast with the local view, the LAB reference. Neither dark matter nor dark energy is required by this model. With an initial constant speed of light during a short time we get inflation (an exponential expansion). This converts, during the inflation time, the Planck's fluctuation length of $10^{-33} \mathrm{~cm}$ to the present size of the Universe (about $10^{28} \mathrm{~cm}$, constant from then on). Thereafter the Mass-Boom takes care to bring the initial values of the Universe (about $10^{15} \mathrm{gr}$ ) to the value at the present time of about $10^{55}$ gr.
\end{abstract}

Keywords Hubble $\cdot$ Speed of light - Anomalous acceleration · Inflation $\cdot$ Cosmology

\section{Introduction}

In 1972 Weinberg presented a relation that contained quantum parameters together with the Hubble cosmological parameter $H$. The order of magnitude of the mass of the fundamental particles was predicted and the relation is still catalogued as "unexplained". It has also been classified as a coincidence of numerical numbers and some explanations (Sidharth 1999) have been proposed, none of them gaining general acceptance. Here we prove it using Newton's universal law of gravitation, together with the momentum conservation law. We take a Machean approach considering the total momentum content of a mass $M$ as $M c$, a constant. The reality of the mass $M$, or the total momentum content $M c$, is due to its interaction with the rest of the Universe. The total relativistic energy $M c^{2}$ is also due to the interaction with the rest of the Universe. With these ideas in mind, together with a time varying speed of light as we will see, we derive the Weinberg relation as a consequence of Newton's laws.

By using a wave to particle interaction technique presented elsewhere (Adams 1982, 1983; Alfonso-Faus 1986) we derive a generalized red shift law that contemplates time variations in $c$ and $G$ (the gravitational "constant"). Then we prove that the speed of light $c$ is proportional to the Hubble parameter $H$, and therefore time-varying. This time variation explains the anomalous acceleration found in space probes like the Pioneer 10/11. With the propagation equation of particles and photons we prove two very important constancies: all gravitational radii are constant. In particular 
the gravitational radius of the Universe is constant. Also the cosmological scale factor of the Universe is constant: neither expansion nor contraction is present in the Universe at a cosmological scale.

The time variation of $c$ implies a time variation of any mass. This is a result of the constancy of momentum: since the relation of any speed $v$ to the speed of light $c, v / c$, has to be a constant to preserve special relativity, the constancy of momentum $m v$ implies the constancy of $m c$. Then a decreasing speed of light implies an increasing mass, a MassBoom as we call it.

Finally we make use of the Einstein cosmological equations to find the cosmological model that this theory predicts: a closed, finite, unlimited, and curved $(k=1)$ Universe with zero cosmological constant and a baryon matter content given by $\Omega_{m}=\Omega_{k}$. Neither dark matter nor dark energy is necessary in this model. However, an initial inflationary phase is also built in the model that brings the initial Planck fluctuation to the present size of the Universe.

\section{Weinberg's relation}

In 1972 Weinberg discussed a relation obtained by using $G$ (gravitation), $c$ (relativity), $\hbar$ (quantum mechanics) and $H$ (cosmology) to arrive at the mass $m$ of a fundamental particle:

$m^{1 / 3} \approx \frac{\hbar^{2} H}{G C}$

We will follow a Machean approach by considering that the maximum momentum content of a mass $M$ is $M c$, a constant due to the rest of the Universe. It has acquired this momentum during a time $t$. Then the average force that can exert is $M c / t$. The result of this heuristic argument can also be obtained by considering the rate of change with time of the total relativistic energy of the mass $M, M c^{2}$, divided by $c$ :

$\frac{1}{c} \frac{d M c^{2}}{d t}=M \frac{d c}{d t}$

We later will prove that the speed of light $c$ is proportional to the Hubble parameter $H, c=H L$, where $L$ is a constant. For a linearly expanding Universe the time derivative of $H$ is $-H^{2}$ so that in absolute value we have for (2)

$M \frac{d c}{d t}=-M H^{2} L=-M H c$

Here we note that the Hubble parameter can be taken, in certain cases, as an operator that obtains the derivative, with respect to the cosmological time, of the function after it (and changing its sign). To obtain the cosmological change with time we just put $H$ in front of the function and multiply by -1 . This average force (3) can be thought of as an action fluxing over a spherical surface of radius $r$ centred on the centre of gravity of $M$. It will act upon the small area represented by $m, m \ll M$, that has a size of the order of its Compton wavelength. Then $m$ will feel a force exerted by the presence of $M$ and given by

$-\frac{G M m}{r^{2}}=-M H c \frac{(\hbar / m c)^{2}}{4 \pi r^{2}}$

and rearranging we arrive at

$4 \pi m^{3}=\frac{\hbar^{2} H}{G c}$

which is the Weinberg's relation (1) within a multiplying factor of less than 3 in front of it.

\section{Generalized red shift law}

In 1983 Adams presented a wave to particle technique to derive the matter and photon propagation. Later, AlfonsoFaus (1986), applied this technique to the case of a timevarying gravitational constant $G$. Now we do the same but allowing the speed of light $c$ to vary with cosmological time $t$ too. We take a spatially flat Friedmann Robertson-Walker metric here. Since the factor in the Einstein's field equations in front of the stress-energy tensor is $G / c^{4}$, we have now the generalized red shift law equation as

$G / c^{4} N_{\gamma} \hbar \nu R=\mathrm{constant}$

Taking into account that the number of photons in a proper volume $N_{\gamma}$ is constant (no particle creation), and using $v=c / \lambda$, we get eliminating $G$ from (5) and (6)

$H N_{\gamma} R(\hbar / m c)^{3}=c \lambda$

The wavelength $\lambda$ is proportional to the cosmological scale factor $R$, and the Compton wavelength $\hbar / m c$ is constant. Then we have

$c=H \times$ constant $=H L$

where $L \approx 10^{28} \mathrm{~cm}$, is the present size of the visible Universe ct. Since the Hubble parameter $H$ is inversely proportional to the cosmological time $t$ so is the speed of light $c$. Substituting (8) into (1) we get the new Weinberg's relation

$m^{3} \approx \frac{\hbar^{2}}{G L}$

Here we see that we have obtained a relation that classically, with $G, m, \hbar$ and $L$ all constants, has no time dependence 
in it. But allowing $G$ and $m$ to vary with time we have for $\hbar$ and $L$ constant

$G m^{3}=$ constant $=\hbar^{2} / L$

This is what is observed in the lunar range experiments with laser. Now, if we demand that the field equations of Einstein's general relativity continue to be derived from the action principle, we need to maintain constant the factors in front of the action integrals. This means

$G / c^{3}=$ constant and $m c=$ constant

And these relations satisfy Weinberg's relation in the form given in (9).

\section{Explanation of the pioneer $10 / 11$ anomalous acceleration}

The fact that the speed of light varies with time as in (8) has enormous cosmological implications. We can get the photon acceleration as

$d c / d t=L d H / d t$

In the laboratory the speed of light is taken as constant $c_{0}$. Then we see a linear expansion of the Universe as $R=c_{0} t$. Since $H$ is $R^{\prime} / R$ we get for the photon acceleration a

$a=d c / d t=-L H^{2}=-H c$

Taking a range of values for $H$ from 60 to $75 \mathrm{Km} / \mathrm{s} / \mathrm{Mpc}$, the theoretical acceleration (13) for the photons is

$a=-(7.13 \pm 0.3) \mathrm{cm} / \mathrm{seg}^{2}$

The observed anomalous acceleration in the Pioneer 10/11 case (Anderson et al. 1998) is

$a_{\mathrm{p}}=-(8.74 \pm 1.33) \mathrm{cm} / \mathrm{seg}^{2}$

which is within the margin given in (14). From this point of view the observed acceleration is not satellite acceleration (as due to gravitational attraction). It is in the photons.

\section{The constancy of gravitational radii}

With the wave to particle technique (Alfonso-Faus 1986) we get now for the matter propagation equation

$$
\left(\left(\frac{G}{c^{2}} N P^{\mu}\right)_{; \alpha}\right) P^{\alpha}=0
$$

Using $P^{\mu}=m U^{\mu}$ we get

$U_{; \alpha}^{\mu} U_{\alpha}+\frac{d \ln \left(\frac{G}{c^{2}} m N\right)}{d t} U^{\mu} U^{0}=0$

and contracting with $U_{\mu}$ we finally get

$\frac{d \ln \left(\frac{G}{c^{2}} m N\right)}{d t}=0 \quad$ i.e. $\frac{G m N}{c^{2}}=$ constant.

This means that all the gravitational radii of masses $m N$ inside its proper volume are constant. The possible expansion or contraction of the Universe does not affect them.

\section{The constancy of the cosmological scale factor}

We have derived elsewhere (Belinchón and Alfonso-Faus 2001) the expression for the zero value of the right hand side of the Einstein's field equations

$\nabla\left(G / c^{4} \cdot T^{\mu \nu}\right)=0$

which is

$\frac{\rho^{\prime}}{\rho}+3(\omega+1) H+\frac{\Lambda^{\prime} c^{4}}{8 \pi G \rho}+\frac{G^{\prime}}{G}-4 \frac{c^{\prime}}{c}=0$

where we have allowed for the time dependence of $G, c$ and $\Lambda$. The cosmological parameter $\Lambda$ is a real constant of integration so that it disappears from (20). In fact we will later show that $\Lambda$ is zero in the cosmological view. Here $\rho$ is the energy density. Using the equation of state $p=w \rho$, integration of (20) gives

$\frac{G \rho}{c^{4}} R^{3(\omega+1)}=\mathrm{constant}$

or equivalently

$G M / c^{2} \cdot R^{3 w}=$ constant

This result is very important. Since all the gravitational radii are constants and if $w$ is not zero, we arrive at the conclusion that the Universe does not expand nor contracts, i.e.

$R=$ constant

Then the gravitational radius of the Universe can be equated to its visible size with $R=c t=$ constant $=L$. For $M$ the mass of the Universe at present we have

$G M / c^{2}=R=c t=L=\mathrm{constant} \approx 10^{28} \mathrm{~cm}$

No length in the Universe is expanding nor contracting. The dynamo paradox, that could ideally be constructed to obtain work from a rod attached to a galaxy that is going away from us, just goes away. 
There is a way to interpret Mach's principle: the rest energy of any mass $m$ is equal to its gravitational potential energy due to the rest of the mass of the Universe:

$G M m / R=m c^{2} \quad$ or $\quad G M / c^{2}=R$

Comparing (24), (25) and (22) we get the value of $w$ as

$W=-1 / 3 \quad$ so that $\quad p=-1 / 3 \rho$

We note that this is the same as the equation of state for photons, with a minus sign. We interpret it as the negative pressure of the gravity quanta.

\section{Mass-Boom and the cosmological equations}

From (11) and (24) we get again the Mass-Boom, effect a linear increase of mass with cosmological time, $M \alpha t$. We take the number of particles in the Universe $N$ as constant so that in a certain system of units we have:

$M=N m=N t$

And from (24) we arrive at

$G / c^{3}=1 / N \quad$ and $\quad m=t$

The Einstein cosmological equations, with $R=$ constant are then

$8 \pi \frac{G p}{c^{2}}+\frac{K c^{2}}{R^{2}}=\Lambda c^{2}$

$-\frac{8 \pi}{3} G \rho_{m}+\frac{K c^{2}}{R^{2}}=\frac{\Lambda c^{2}}{3}$

Now with $w=-1 / 3 \Lambda$ has to be identically zero. Then the two cosmological equations reduce to just one, i.e.

$\frac{K c^{2}}{R^{2}}=\frac{8 \pi}{3} G \rho_{m}$

We see that $K=1$ is the solution to this equation. This cosmological model is spherical, closed, finite, unlimited, and static. Equation (30) can be interpreted as equilibrium between centrifugal forces, represented by the curvature term, and the negative pressure, the gravitational attraction.

In the usual dimensionless parameters $\Omega$ we have the equivalent to (30)

$\Omega_{m}=\Omega_{k} \quad$ (undetermined)

We can take the value 0.05 for both parameters. Then neither dark matter nor dark energy is necessary in this model.

\section{The initial conditions of the universe}

During the first instants of time the speed of light must have been almost constant. This means that the Hubble parameter then was constant so that we have at that time:

$H=R^{\prime} / R=\mathrm{constant}$

Integrating the above relation we get the solution:

$R=R_{0} e^{H t}$

This exponential expansion is equivalent to the very well known inflation phase at the initial stages of the Universe. During the inflation phase the first Planck's fluctuation of size $10^{-33} \mathrm{~cm}$ expanded very rapidly to the size $10^{28} \mathrm{~cm}$, as of today, so that we have

$e^{H t}=10^{61}$

If $t_{1}$ is the first tic of the Universe, and $t_{i}$ is the duration of the inflationary phase, then

$t_{i} / t_{1}=H t=61 \ln 10 \approx 140$

Hence, only 140 tics were necessary to inflate the Planck's fluctuation to the present size of the Universe, and thereafter it remained at constant size. In our theory Planck's units have a time dependence as

$\left(\frac{\hbar c}{G}\right)^{1 / 2}=10^{60} / c=10^{20} t \approx 10^{-5} \mathrm{gr}$,

$\left(\frac{G \hbar}{c^{3}}\right)^{1 / 2}=\mathrm{constant}=1 / 10^{20} \approx 10^{-33} \mathrm{~cm}$,

$\left(\frac{G \hbar}{c^{5}}\right)^{1 / 2}=1 /\left(10^{20} c\right)=t / 10^{60} \approx 10^{-44} \mathrm{~s}$

These units had an initial value of

$t=10^{40}$

$t_{1}=1$,

$\left(\frac{\hbar c}{G}\right)_{1}^{1 / 2}=10^{20} t_{1} \approx 10^{-45} \mathrm{gr}$,

$\left(\frac{G \hbar}{c^{3}}\right)_{1}^{1 / 2} \approx 10^{-33} \mathrm{~cm}=$ constant

$\left(\frac{G \hbar}{c^{5}}\right)_{1}^{1 / 2}=t_{1} / 10^{60} \approx 10^{-83} \mathrm{~s}$

Here we see that inflation by a factor of $10^{61}$ gives an initial stage of the Universe as

$M_{i}=10^{15} \mathrm{gr}, \quad L=10^{28} \mathrm{~cm}=$ constant,

$t_{i}=10^{-23} \mathrm{~s}$ 
From then on we have $5 \times 10^{40}$ tics to arrive at

$M_{0}=5 \times 10^{55} \mathrm{gr}, \quad L=10^{28} \mathrm{~cm}=\mathrm{constant}$,

$t_{0}=5 \times 10^{17} \mathrm{~s}$

which is the present state of the Universe.

\section{Conclusions}

The wave-to-particle interaction technique is a powerful tool to determine very important relations in Nature. We have found the matter and photon propagation equations using the Einstein's general relativity field equations. We have found a generalized red shift law. We have allowed for time-varying $G, m$ and $c$. We have found the new constants of Nature $G / c^{3}, m c$ and $c / H$. We have defined a Mass-Boom cosmological model, a new frame of work for cosmology that has a decreasing speed of light and an increasing mass with cosmological time. The predicted photon acceleration agrees well with the anomalous acceleration of the Pioneer 10/11 satellites.

\section{References}

Adams, P.J.: Large numbers hypothesis. I. Classical formalism. Int. J. Theor. Phys. 21, 603 (1982)

Adams, P.J.: Large numbers hypothesis. II. Electromagnetic radiation. Int. J. Theor. Phys. 22, 421 (1983)

Alfonso-Faus, A.: Cosmology with time-varying $G$. Int. J. Theor. Phys. 25(3), 293-316 (1986)

Anderson, J.D., et al.: Indication, from Pioneer 10/11, Galileo, and Ulysses data, of an apparent anomalous, weak, long-range acceleration. Phys. Rev. Lett. 81, 2258 (1998)

Belinchón, J.A., Alfonso-Faus, A.: A theory of time-varying constants. Int. J. Mod. Phys. D 10, 299-310 (2001). arXiv:gr-qc/0404044

Sidharth, B.G.: The Universe of Chaos and Quanta, 8 February 1999. arXiv:qant-ph/9902028

Weinberg, S.: Gravitation and Cosmology. Wiley, New York (1972) 